\title{
Características e deficiências dos programas de pós-graduação em oftalmologia no Brasil segundo pós-graduandos participantes
}

\author{
Caracteristics and deficiencies of ophthalmology \\ residency programs in Brazil: residents evaluation
}

Camila Vieira Oliveira Carvalho Ventura', Mirela Luna Santana Gomes², Bruna Vieira Oliveira Carvalho Ventura $^{3}$, Liana Oliveira Ventura ${ }^{4}$, Carlos Teixeira Brandt ${ }^{5}$

\begin{abstract}
RESUMO
Objetivo: Analisar a percepção dos pós-graduandos participantes quanto à qualidade dos cursos lato sensu de oftalmologia do Brasil e apontar as áreas que necessitam melhorias. Métodos: Realizou-se um estudo transversal e descritivo com 107 pós-graduandos de oftalmologia de cursos credenciados pelo Ministério da Educação e Cultura (MEC) e/ou Conselho Brasileiro de Oftalmologia (CBO). Avaliou-se através de questionário as preceptorias (clínica, cirúrgica e emergência), as necessidades de melhoria curricular, aulas teóricas, cirurgias experimentais, atividades cirúrgicas e infraestrutura das instituições participantes. Resultados: Vinte e seis pós-graduandos $(24,3 \%)$ estudavam em instituições credenciadas pelo MEC, 25 (23,4\%) pelo CBO e 56 (52,3\%) pelo CBO e MEC. A qualidade da preceptoria clínica, cirúrgica e na emergência mostrou-se satisfatória (86,0\%,87,0\% e 67,3\% respectivamente). As preceptorias clínica, cirúrgica e na emergência estiveram presentes em todos os turnos em $55,1 \%$, $56,1 \%$ e $36,4 \%$, respectivamente. A maioria dos alunos deseja maior oferta de cirurgias experimentais $(75,7 \%)$, melhoria de recursos para o ensino (63,6\%), dos equipamentos (74,8\%) e das instalações físicas (67,3\%). Conclusão: As variáveis analisadas foram similares nos serviços credenciados pelo $\mathrm{MEC}, \mathrm{CBO}$ e por ambos. A qualidade das preceptorias nos serviços era satisfatória, embora não eram presenciais em todos os turnos em várias instituições. Os alunos sugeriram um aumento do volume cirúrgico e de cirurgias experimentais e melhoria na infraestrutura.

Descritores: Programas de pós-graduação em Saúde;Treinamento; Oftalmologia/educação; Currículo; Internato e Residência
\end{abstract}

\footnotetext{
${ }^{1}$ Médica, aluna do primeiro ano do Curso de Especialização em Oftalmologia da Fundação Altino Ventura - FAV - Recife (PE), Brasil; ${ }^{2}$ Médica, aluna do terceiro ano do Curso de Especialização em Oftalmologia da Fundação Altino Ventura Fundação Altino Ventura FAV - Recife (PE), Brasil;

${ }^{3}$ Médica, aluna do segundo ano do Curso de Especialização em Oftalmologia Fundação Altino Ventura - FAV - Recife (PE), Brasil; ${ }^{4}$ Departamento de Oftalmologia Pediátrica e Estrabismo do Hospital de Olhos de Pernambuco (HOPE); Fundação Altino Ventura FAV - Recife (PE), Brasil;

${ }^{5}$ Coordenador da pós-graduação da UFPE. Coordenador do Departamento de Investigação Cientifica da Fundação Altino Ventura FAV - Recife ( PE), Brasil.
}

Os autores declaram inexistir conflitos de interesse

Recebido para publicação em 18/5/2011 - Aceito para publicação em 15/9/2011 


\begin{abstract}
Purpose: Analyze residents' perception on ophthalmology training in Brazil by classifying the quality of attendings' supervision. Also, identify areas that need better investment.Methods: This was a descriptive and transversal study that analyzed residents' opinion about Brazilian institutions accredited by the Ministry of Education and Culture (MEC) or by the Brazilian Council of Ophthalmology (CBO) or by both. A multiple-choice survey was applied to 107 residents to evaluate attendings' (clinical, surgical and in the emergency room) supervision, and the need of improvement in different areas such as, curriculum, amount/quality of surgical procedures, wetlabs and infrastructure. Results: Twenty-six residents (24.3\%) were from institutions accredited by MEC, 25 (23.4\%) by CBO, and 56 (52.3\%) by both, MEC and CBO. Attendings' supervision quality was considered satisfactory during surgeries $(86,0 \%)$, clinical examinations $(87,0 \%)$ and in the emergency room $(67.3 \%)$. Full-time supervision was present in these departments in $55.1 \%, 56.1 \%$, and $36.4 \%$, respectively. Wetlabs, resources, equipments, and physical facilities were pointed as areas that need investment in $75.7 \%, 63.6 \%, 74.8 \%$, and $67.3 \%$, respectively. Conclusion: This study showed no statistical relevance between the different institutions' accreditation and the variables analyzed. In general, direct supervision in Brazilian institutions is satisfactory, although many are not full-time. Residents suggested increasing the number of surgeries and wetlabs, and investing in the institutions' infrastructure.
\end{abstract}

Residency

Keywords: Health Postgraduate Programs; Training; Ophthalmology/education; Curriculum; Internship and

\section{INTRODUÇÃO}

A oftalmologia, especialidade clínico-cirúrgica adquiriu sua autonomia como entidade médica em meados de 1950, quando a educação e a prática oftalmológica e otorrinolaringológica se distinguiram..$^{(1-3)}$

O ensino da oftalmologia nos Estados Unidos (EUA) segue critérios específicos determinados pelo Conselho de Acreditação para Educação em Graduação Médica (ACGME), que visa uma padronização curricular e um sistema de avaliação das residências médicas. Há pouco menos de quatro anos, esse currículo passou a obedecer a seis competências gerais, incluindo o cuidado ao paciente, conhecimento médico, profissionalismo, comunicação, habilidade interpessoal e o sistema de aprendizagem (systems-based learning). Desde 1978, as Residências Médicas americanas são rigorosamente controladas pelo Comitê de Revisão de Residência (RRC), que para a subespecialidade da oftalmologia é composta por nove membros da American Board of Ophthalmology, American Academy of Ophthalmology e American Medical Association. ${ }^{(4-8)}$

No Canadá, os programas de pós-graduação são reconhecidos internacionalmente pelos seus altos padrões, sendo credenciados e regulamentados pelo Royal College of Physicians and Surgeons of Canada (RCPSC), que segue as diretrizes do International Council of Ophthalmology $(\text { ICO })^{(9)}$
No Brasil, existem dois órgãos independentes responsáveis pelo credenciamento e supervisão dos cursos de pós-graduação Lato Sensu em oftalmologia: a Comissão Nacional de Residência Médica (CNRM) do Ministério da Educação e Cultura (MEC) e a Associação Médica Brasileira (AMB) através do Conselho Brasileiro de Oftalmologia (CBO). Ambas têm como atribuições a educação continuada, através de eventos científicos e a padronização do ensino oftalmológico nos cursos credenciados. O CBO, através da sua comissão de ensino organiza a Prova Nacional de Oftalmologia, concedendo aos aprovados o Título de Especialista em Oftalmologia. Tal competência era exclusividade do CBO até a década de oitenta, quando o MEC oficialmente adquiriu esta autonomia. ${ }^{(10-11)}$

Nos últimos tempos, os órgãos de credenciamento têm buscado um aperfeiçoamento e unificação do currículo nas instituições de ensino em oftalmologia com o objetivo de uniformizar a qualidade do ensino no país, acompanhar os avanços tecnológicos, sem perder o padrão de uma medicina humanizada. ${ }^{(12,13)}$ Informações básicas para a construção do conhecimento e subsídios como recursos humanos e infraestrutura para a boa formação do profissional em oftalmologia vem sendo discutidos amplamente. . $^{(7,14-16)}$

Objetivou-se nesse estudo analisar a qualidade dos cursos de oftalmologia no Brasil, com foco maior no suporte ao ensino durante a pós-graduação e as áreas que necessitam melhorias nas instituições de ensino no Brasil, levando em consideração a opinião dos pós- 
graduandos, uma vez que não foi encontrado nenhum trabalho brasileiro, até o momento, com esse enfoque.

\section{Métodos}

Realizou-se um estudo observacional e analítico baseado nas respostas dos questionários fechados aplicados aos pós-graduandos de oftalmologia do curso de residência médica credenciados pelo MEC e aos do curso de especialização credenciados pelo CBO. A coleta de dados foi realizada durante o XVIII Congresso Brasileiro de Prevenção a Cegueira e Reabilitação Visual realizado em Florianópolis em 2008.

O questionário era composto de 22 perguntas com dados de identificação (idade, instituição onde cursava a pós-graduação, credenciamento da instituição, localização); avaliação quantitativa e qualitativa concernentes à percepção dos alunos quanto às preceptorias (clínica, cirúrgica e emergência oftalmológica) nas instituições; necessidades de melhoria curricular; aulas teóricas; cirurgias experimentais; atividades cirúrgicas; e infraestrutura (biblioteca/internet, equipamentos e instalações físicas).

O estudo teve a participação de 107 oftalmologistas em formação, que cursavam do primeiro ao terceiro ano, em 45 instituições brasileiras, sendo seu preenchimento voluntário e anônimo. Para fins analíticos, os dados foram divididos em três grupos de acordo com a instituição a qual o aluno estava vinculado: $\mathrm{CBO}$; $\mathrm{MEC}$; $\mathrm{CBO}$ e MEC. Considerou-se como qualidade de preceptoria satisfatória, aquela em que o aluno qualificou como excelente/boa e insatisfatória a classificada como regular/ausente.

O projeto do estudo foi aprovado pelo Comitê de Ética em Pesquisa em Seres Humanos da Fundação Altino Ventura. Os alunos dos cursos de pós-graduação em oftalmologia foram orientados quanto à natureza do estudo e convidados a participar do mesmo, procedendo à assinatura do Termo de Consentimento Livre e Esclarecido.

Os resultados foram expressos por suas frequências absolutas e relativas, sendo realizado uma análise através do teste Qui-quadrado.

\section{$\underline{\text { Resultados }}$}

Cento e sete questionários preenchidos pelos pósgraduandos atenderam aos critérios adotados para este estudo. A idade dos participantes variou de 24 a 33 anos, com média de 26,8 $\pm 1,9$ anos. Vinte e seis pós-graduandos

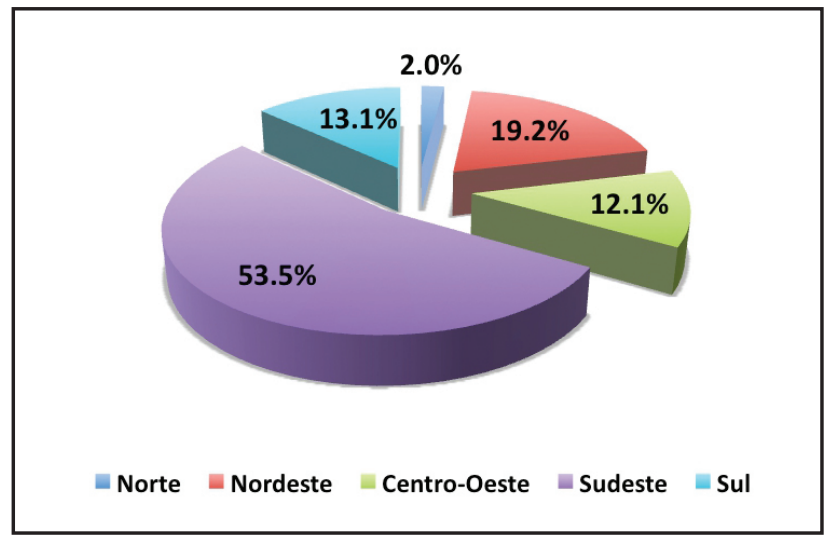

Figura 1: Distribuição regional por frequência das instituições brasileiras de ensino em oftalmologia

$(24,3 \%)$ estudavam em instituições de ensino da oftalmologia credenciadas pelo MEC, 25 (23,4\%) pelo CBO e $56(52,3 \%)$ pelo CBO e MEC.

A maior concentração dos alunos estudava em cursos da região sudeste do Brasil (53,5\%) (Figura 1).

Os alunos referiram preceptoria clínica presencial em 105 (98,1\%) serviços, estando presentes em todos os turnos (manhã e tarde) em 59 (55,1\%) das instituições avaliadas. A qualidade da preceptoria clínica mostrouse satisfatória na opinião de $92(86,0 \%)$ pós-graduandos. Preceptoria cirúrgica foi referida em 100 (93,5\%) instituições, atuando de forma presencial em todos os turnos (manhã e tarde) em $60(56,1 \%)$ dos serviços, com qualidade satisfatória em $93(87,0 \%)$.

Não houve diferença estatisticamente significante de frequência dos alunos credenciados pelo $\mathrm{CBO}, \mathrm{MEC}$ e $\mathrm{CBO}$ e MEC em relação a presença de preceptoria clínica, em todos os turnos e a sua qualidade (teste de Qui-quadrado p-valor= 0,5723; 0,9189 e 0,2584, respectivamente). Tal tendência também foi verificada na preceptoria cirúrgica (teste Qui-quadrado, $\mathrm{p}=0,5784$, $\mathrm{p}=0,4519$ e $\mathrm{p}=0,6304$, respectivamente) (Tabelas 1 e 2)

Preceptoria nas emergências oftalmológicas esteve presente em $70(65,4 \%)$ instituições, não havendo preceptoria em todos os turnos em $68(63,6 \%)$ serviços, especialmente naqueles credenciados pelo $\mathrm{CBO}(72,0 \%)$ e pelo CBO e MEC (62,5\%). Esta preceptoria foi apontada como de menor qualidade, especialmente nas instituições credenciadas pelo MEC, onde 11 (42,3\%) foi classificada como insatisfatória. Não houve diferença estatisticamente significante entre os órgãos de credenciamento (CBO, MEC e CBO e MEC) em relação a presença de preceptoria na emergência, em todos os turnos e a sua qualidade (teste Qui-quadrado $\mathrm{p}=$ $0,6304, p=0,5537$ e $p=0,3757$ ) (Tabela 3). 
Tabela 1

Distribuição das informações dos pós-graduandos quanto à preceptoria clínica segundo a instituição de credenciamento (CBO, MEC, CBO e MEC) por frequência

\begin{tabular}{|c|c|c|c|c|c|c|c|c|c|c|c|c|}
\hline & \multicolumn{4}{|c|}{$\begin{array}{c}\text { Presença da } \\
\text { Preceptoria Clínica }\end{array}$} & \multicolumn{4}{|c|}{ Todos os turnos } & \multicolumn{4}{|c|}{ Qualidade } \\
\hline & \multicolumn{2}{|c|}{ Sim } & \multicolumn{2}{|c|}{ Não } & \multicolumn{2}{|c|}{ Sim } & \multicolumn{2}{|c|}{ Não } & \multicolumn{2}{|c|}{ Satisfatório } & \multicolumn{2}{|c|}{ Insatisfatório } \\
\hline & $\mathbf{n}$ & $\%$ & $\mathbf{n}$ & $\%$ & $\mathbf{n}$ & $\%$ & $\mathbf{n}$ & $\%$ & $\mathbf{n}$ & $\%$ & $\mathbf{n}$ & $\%$ \\
\hline $\mathrm{CBO}$ & 24 & 96,0 & 1 & 4,0 & 13 & 52,0 & 12 & 48,0 & 19 & 76,0 & 6 & 24,0 \\
\hline MEC & 26 & 100,0 & 0 & 0,0 & 15 & 57,7 & 11 & 42,3 & 23 & 88,5 & 3 & 11,5 \\
\hline $\mathrm{MEC}$ e $\mathrm{CBO}$ & 55 & 98,2 & 1 & 1,8 & 31 & 55,4 & 25 & 44,6 & 50 & 89,3 & 6 & 10,7 \\
\hline Total & 105 & 98,1 & 2 & 1,9 & 59 & 55,1 & 48 & 44,9 & 92 & 86,0 & 15 & 14,0 \\
\hline p-valor & \multicolumn{4}{|c|}{0,5723} & \multicolumn{4}{|c|}{0,9189} & \multicolumn{4}{|c|}{0,2584} \\
\hline
\end{tabular}

Tabela 2

Distribuição das informações dos pós-graduandos quanto à preceptoria cirúrgica segundo a instituição de credenciamento (CBO, MEC, CBO e MEC) por frequência

\begin{tabular}{|c|c|c|c|c|c|c|c|c|c|c|c|c|}
\hline & \multicolumn{4}{|c|}{$\begin{array}{c}\text { Presença da } \\
\text { Preceptoria Clínica }\end{array}$} & \multicolumn{4}{|c|}{ Todos os turnos } & \multicolumn{4}{|c|}{ Qualidade } \\
\hline & \multicolumn{2}{|c|}{ Sim } & \multicolumn{2}{|c|}{ Não } & \multicolumn{2}{|c|}{ Sim } & \multicolumn{2}{|c|}{ Não } & \multicolumn{2}{|c|}{ Satisfatório } & \multicolumn{2}{|c|}{ Insatisfatóric } \\
\hline & $\mathbf{n}$ & $\%$ & $\mathbf{n}$ & $\%$ & $\mathbf{n}$ & $\%$ & $\mathbf{n}$ & $\%$ & $\mathbf{n}$ & $\%$ & $\mathbf{n}$ & $\%$ \\
\hline $\mathrm{CBO}$ & 24 & 96,0 & 1 & 4,0 & 12 & 48,0 & 13 & 52,0 & 21 & 84,0 & 4 & 16,0 \\
\hline MEC & 25 & 96,2 & 1 & 3,8 & 17 & 65,4 & 9 & 34,6 & 24 & 92,4 & 2 & 7,6 \\
\hline $\mathrm{MEC}$ e $\mathrm{CBO}$ & 51 & 91,0 & 5 & 9,0 & 31 & 55,4 & 25 & 44,6 & 48 & 85,8 & 8 & 14,2 \\
\hline Total & 100 & 93,5 & 7 & 6,5 & 60 & 56,1 & 47 & 43,9 & 93 & 87,0 & 14 & 13,0 \\
\hline p-valor & \multicolumn{4}{|c|}{0,5784} & \multicolumn{4}{|c|}{0,4519} & \multicolumn{4}{|c|}{0,6304} \\
\hline
\end{tabular}

Tabela 3

Distribuição das informações dos pós-graduandos quanto à preceptoria da emergência segundo a instituição de credenciamento (CBO, MEC, CBO e MEC) por frequência

\begin{tabular}{|c|c|c|c|c|c|c|c|c|c|c|c|c|}
\hline & \multicolumn{4}{|c|}{$\begin{array}{c}\text { Presença da } \\
\text { Preceptoria Clínica }\end{array}$} & \multicolumn{4}{|c|}{ Todos os turnos } & \multicolumn{4}{|c|}{ Qualidade } \\
\hline & \multicolumn{2}{|c|}{ Sim } & \multicolumn{2}{|c|}{ Não } & \multicolumn{2}{|c|}{ Sim } & \multicolumn{2}{|c|}{ Não } & \multicolumn{2}{|c|}{ Satisfatório } & \multicolumn{2}{|c|}{ Insatisfatório } \\
\hline & $\mathbf{n}$ & $\%$ & n & $\%$ & $\mathbf{n}$ & $\%$ & $\mathbf{n}$ & $\%$ & $\mathbf{n}$ & $\%$ & $\mathbf{n}$ & $\%$ \\
\hline $\mathrm{CBO}$ & 17 & 68,0 & 8 & 32,0 & 7 & 28,0 & 18 & 72,0 & 19 & 76,0 & 6 & 24,0 \\
\hline MEC & 15 & 57,7 & 11 & 2,3 & 11 & 42,3 & 15 & 57,7 & 15 & 57,7 & 11 & 42,3 \\
\hline $\mathrm{MEC}$ e $\mathrm{CBO}$ & 38 & 67,9 & 18 & 32,1 & 21 & 37,5 & 35 & 62,5 & 38 & 67,9 & 18 & 32,1 \\
\hline Total & 70 & 65,4 & 37 & 34,6 & 39 & 36,4 & 68 & 63,6 & 72 & 67,3 & 35 & 32,7 \\
\hline p-valor & \multicolumn{4}{|c|}{0,6354} & \multicolumn{4}{|c|}{0,5537} & \multicolumn{4}{|c|}{0,3757} \\
\hline
\end{tabular}


Tabela 4

Distribuição das necessidades de aperfeiçoamento curricular segundo a instituição de credenciamento (CBO, MEC, CBO e MEC) por frequência

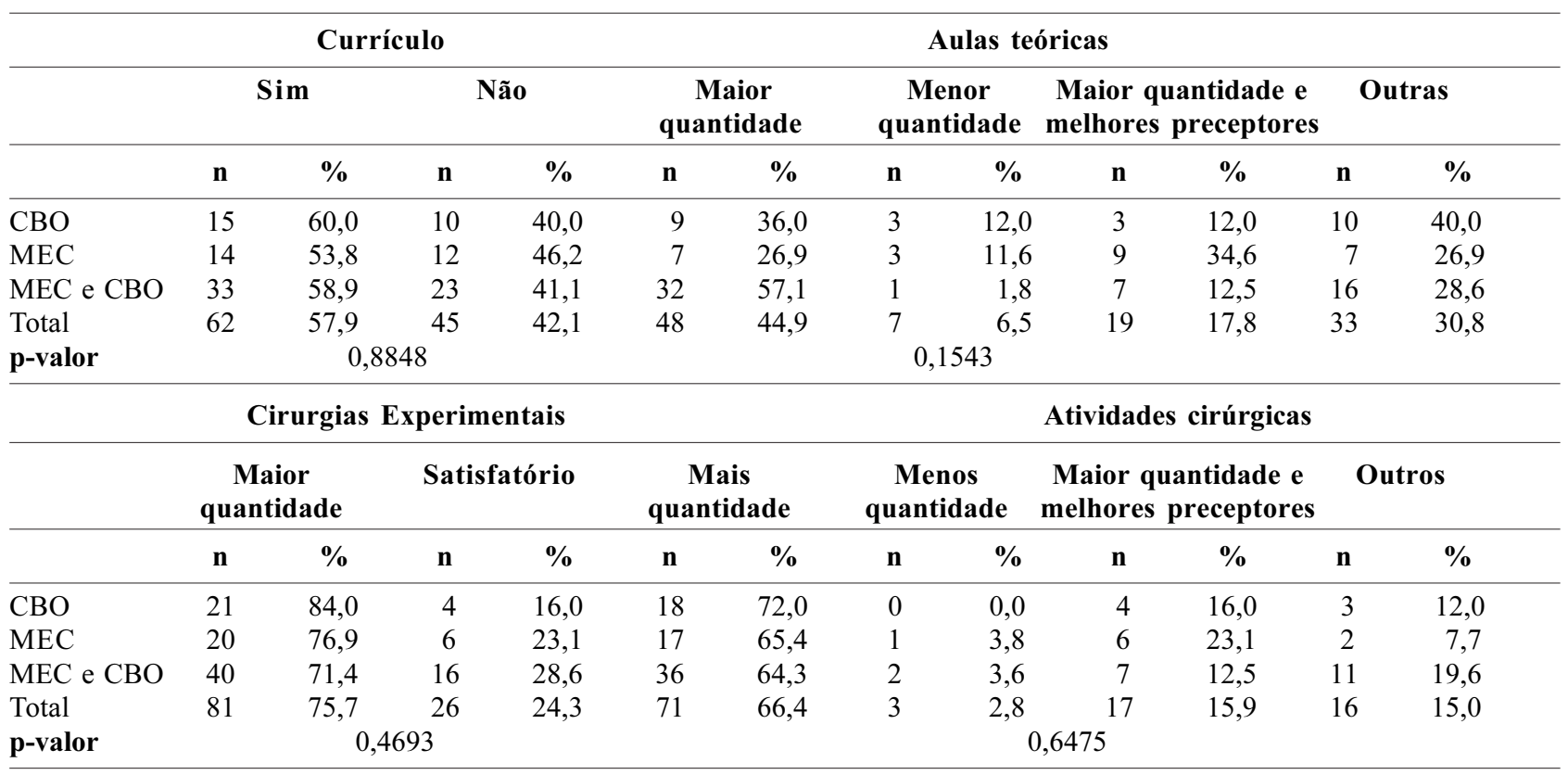

Tabela 5

Distribuição das necessidades de melhoria na infraestrutura segundo a instituição de credenciamento (CBO, MEC, $\mathrm{CBO}$ e MEC) por frequência

\begin{tabular}{|c|c|c|c|c|c|c|c|c|c|c|c|c|}
\hline & \multicolumn{4}{|c|}{ Internet / Biblioteca } & \multicolumn{4}{|c|}{ Equipamentos } & \multicolumn{4}{|c|}{ Instalações Físicas } \\
\hline & \multicolumn{2}{|c|}{ Necessidade } & \multicolumn{2}{|c|}{ Satisfatório } & \multicolumn{2}{|c|}{ Necessidade } & \multicolumn{2}{|c|}{ Satisfatório } & \multicolumn{2}{|c|}{ Necessidade } & \multicolumn{2}{|c|}{ Satisfatório } \\
\hline & $\mathbf{n}$ & $\%$ & $\mathbf{n}$ & $\%$ & $\mathbf{n}$ & $\%$ & $\mathbf{n}$ & $\%$ & $\mathbf{n}$ & $\%$ & n & $\%$ \\
\hline $\mathrm{CBO}$ & 17 & 68,0 & 8 & 32,0 & 19 & 76,0 & 6 & 24,0 & 17 & 68,0 & 8 & 32,0 \\
\hline $\mathrm{MEC}$ & 16 & 61,5 & 10 & 38,5 & 20 & 76,9 & 6 & 23,1 & 18 & 69,2 & 8 & 30,8 \\
\hline $\mathrm{MEC}$ e CBO & 35 & 62,5 & 21 & 37,5 & 41 & 73,2 & 15 & 26,8 & 37 & 66,0 & 19 & 34,0 \\
\hline Total & 68 & 63,6 & 39 & 36,4 & 80 & 74,8 & 27 & 25,2 & 72 & 67,3 & 35 & 32,7 \\
\hline p-valor & \multicolumn{4}{|c|}{0,8668} & & & \multicolumn{2}{|c|}{0,925} & \multicolumn{4}{|c|}{0,957} \\
\hline
\end{tabular}

Os alunos revelaram uma necessidade de melhoria do conteúdo nuclear programático do currículo na maioria das instituições avaliadas, não havendo significância estatística entre o tipo do credenciamento (teste Qui-quadrado $\mathrm{p}=0,8848$ ). Foi sugerido pelos alunos maior quantidade de aulas teóricas em 48 (44,9\%) dos casos e maior volume cirúrgico em $71(66,4 \%)$ (Tabela 4).

Maior necessidade de cirurgias experimentais foi sugerida por 81 alunos $(75,7 \%)$, especialmente nas ins- tituições credenciadas pelo CBO $(84,0 \%)$, entretanto não houve significância estatística entre os grupos estudados (teste Qui-quadrado $\mathrm{p}=0,4693$ ). Além disso, melhoria de recursos para o ensino (biblioteca / internet) foi indicada por 68 alunos $(63,6 \%)$, dos equipamentos e instalações físicas por $80(74,8 \%)$ e $72(67,3 \%)$ alunos, respectivamente (Tabela 4). Não houve significância estatística entre o órgão de credenciamento e as infraestruturas analisadas (teste Qui-quadrado, $\mathrm{p}=0,8668$, $\mathrm{p}=0,925$ e $\mathrm{p}=0,957$, respectivamente) (Tabela 5). 


\section{DiscussÃo}

O papel de assegurar a formação de oftalmologistas éticos, competentes, habilidosos e preparados para o mercado de trabalho tem sido um compromisso constante dos cursos de pós-graduação em oftalmologia do Brasil.

Estudos recentes demonstram a importância da valorização da perspectiva dos pós-graduandos quanto ao ensino da oftalmologia. ${ }^{(2,5,9,12)} \mathrm{O}$ presente estudo foi realizado com este objetivo. Ofereceu-se a oportunidade para que alunos de pós-graduação pudessem avaliar a qualidade e recomendar melhorias para o currículo e infraestrutura das instituições.

Em 1983, havia no país 32 cursos credenciados pelo CBO e 30 pelo MEC. ${ }^{(13)}$ Nos últimos anos, tem se observado um grande aumento da oferta de cursos de pós-graduação em oftalmologia, tanto pelas instituições credenciadas pelo MEC (87), quanto pelas credenciadas pelo CBO (50), sendo que 42 instituições estão credenciadas pelos dois órgãos (MEC e CBO). ${ }^{(10,17)}$ Uma explicação plausível para este crescimento deve-se a necessidade de suprir um país tão populoso, considerado o quinto maior do mundo em termo de dimensões. ${ }^{(18)}$ Entretanto, este crescimento não tem seguido uma distribuição regional homogênea, o que é compatível com os dados obtidos da amostra estudada, onde o sudeste concentrou a maior frequência dos pós-graduandos credenciados pelo MEC $(52,87 \%), \mathrm{CBO}(58,82 \%)$ e por ambos os órgãos simultaneamente $(61,9 \%){ }^{(19)}$

A oferta e distribuição de serviços credenciados pelo CBO ou pelo MEC para o ensino da oftalmologia no país deve ser visto com cautela, no sentido de promover um ensino de qualidade. Verificou-se que existe uma fragmentação na supervisão e credenciamento nos cursos de pós-graduação em oftalmologia do Brasil, devendo haver um maior intercâmbio entre o $\mathrm{CBO}$ e a CNRM. ${ }^{(11)}$ Os dados obtidos no presente estudo também demonstram que na percepção do aluno existe a necessidade de uma melhoria na qualidade do ensino da oftalmologia do Brasil, tanto nas instituições credenciadas pelo CBO quanto pelo MEC. Alguns serviços não oferecem preceptoria clínica, cirúrgica e na emergência oftalmológica em todos os turnos, falhando também quanto à qualidade do ensino prestado. Estes fatores podem causar sérios prejuízos na curva de aprendizado do médico ainda em formação, por isso o investimento nos recursos humanos deve ser prioridade nas instituições, pois são responsáveis pela transmissão do conhecimento e sua deficiência pode repercutir negativamente na soci- edade, gerando profissionais inseguros na conduta de seus pacientes. ${ }^{(3,7-9,12)}$

A necessidade de melhoria do conteúdo nuclear programático foi constatada anteriormente, onde mais de $20 \%$ dos cursos não possuíam disciplina de histologia, patologia, imunologia, farmacologia, microbiologia, cirurgia experimental em seus programas. ${ }^{(13)}$ Neste estudo, a maioria dos alunos manifestou o desejo de ter maior acesso a cirurgias experimentais $(75,7 \%)$ e realizar maior volume cirúrgico $(66,4 \%)$. A habilidade cirúrgica individual exerce uma importante influência no desempenho profissional, entretanto a prática cirúrgica experimental aliada a um maior número de procedimentos realizados sob supervisão minimiza as taxas de complicações operatórias. ${ }^{(9,20)}$ Melhor infraestrutura das instituições de ensino (equipamentos, acervo da biblioteca, acesso a internet e melhores instalações físicas), pode minimizar de forma significativa as diferenças da saúde ocular da população dos países em desenvolvimento. ${ }^{(12)}$

Preocupados em oferecer um ensino de qualidade, o Conselho Federal de Medicina (CFM) aprovou a resolução 1785/2006 da Comissão Mista de Especialidades (CME), composta pela CFM, AMB e a CNRM, onde foram instituídas normas orientadoras e reguladoras para a padronização das residências médicas de cada especialidade quanto ao currículo. ${ }^{(21)}$ Com esta visão, o CBO, através da comissão de ensino, tem realizado fóruns para os diretores dos 50 cursos credenciados do país. ${ }^{(10)}$ A meta desses fóruns é padronizar um currículo mínimo, obtido com a cooperação das sociedades de cada subespecialidade afiliadas ao CBO. Este currículo deve atender aos critérios internacionais e ao mesmo tempo se adequar à realidade brasileira.

O ensino da oftalmologia nos EUA e Canadá, seguem os critérios determinados pelo RRC e RCPSC, respectivamente. ${ }^{(4-9)}$ Programas virtuais como o "Medical Resident Progress Notebook (RPN)" e o "Surgical RPN" utilizados nos EUA podem ser uma poderosa ferramenta na tentativa de padronização curricular entre os cursos, permitindo uma integração entre as instituições, além de ser fonte complementar de disciplinas básicas dentro da oftalmologia. ${ }^{(11,13)}$

\section{Conclusão}

As variáveis analisadas foram similares nos serviços credenciados pelo MEC, CBO e por ambos.

Em geral, as instituições oferecem as preceptorias (clínica, cirúrgica e na emergência) de qualidade 
satisfatória, no entanto nem todas são presenciais em todos os turnos. A maioria dos pós-graduandos sugeriu um aumento do volume cirúrgico e do número de cirurgias experimentais, melhoria na infraestrutura, dos equipamentos e instalações físicas.

Este estudo é de grande impacto na área do ensino e pode contribuir para a formação de novas políticas de ensino na sociedade brasileira de oftalmologia.

\section{RefERÊNCIAS}

1. Truhlsen SM. The American Academy of Ophthalmology. In: Albert DM, Edward DD, editors. The history of ophthalmology. Cambridge: Blackwell Science; 1996. p.323-58.

2. Pankratz MJ, Helveston EM. Ophthalmology. The resident's perspective. Arch Ophthalmol. 1992;110(1):37-43.

3. Liesegang TJ, Hoskins HD Jr, Albert DM, O'Day DM, Spivey BE, Sadun AA, et al. Ophthalmic education: where have we come from, and where are we going? Am J Ophthalmol. 2003;136(1):114-21. Review.

4. Lee AG, Beaver HA, Greenlee E, Oetting TA, Boldt HC, Olson R, et al. Teaching and assessing systems-based competency in ophthalmology residency training programs. Surv Ophthalmol. 2007;52(6):680-9. Review.

5. McDonnell PJ, Kirwan TJ, Brinton GS, Golnik KC, Melendez RF, Parke DW 2nd, et al. Perceptions of recent ophthalmology residency graduates regarding preparation for practice. Ophthalmology. 2007;114(2):387-91.

6. Trobe JD, Fraunfelder F, Jones DB, Kearns TP, Kinder RS, Milder B, et al. Monitoring ophthalmology graduate education. The Residency Review Committee. Arch Ophthalmol. 1982;100(1):67-71.

7. Lee AG. The new competencies and their impact on resident training in ophthalmology. Surv Ophthalmol. 2003;48(6):65162. Review.

8. An update on the accreditation of graduate education in ophthalmology. The Residency Review Committee for Ophthalmology. Arch Ophthalmol. 1987;105(9):1189-94.

9. Zhou AW, Noble J, Lam WC. Canadian ophthalmology residency training: an evaluation of resident satisfaction and comparison with international standards. Can J Ophthalmol. 2009;44(5):540-7.

10. Conselho Brasileiro de Oftalmologia. Quem somos. [citado 2010 Ago 30]. Disponível em: http://www.cbo.com.br/novo/ medicos/institucional/quem-somos
11. Waisberg Y, Beniz Neto J. Considerações sobre a pósgraduação em oftalmologia no Brasil. Rev Bras Oftalmol. 1984;43(6):211-8.

12. Gogate P, Deshpande M, Dharmadhikari S. Which is the best method to learn ophthalmology? Resident doctors' perspective of ophthalmology training. Indian J Ophthalmol. 2008;56(5):409-12.

13. Lee AG, Koch DD. A standardized curriculum and evaluation system for an ophthalmology residency. Acad Med. 1998;73(5):447-8. Comment on Acad Med. 1997;72(7):564.

14. Delgado AC, Sena M, Corbera JC, Ventura LO. Perfil de 80 pós-graduandos em oftalmologia do Estado de Pernambuco, de 1998 a 2001. Rev Bras Oftalmol. 2002;61(3):204-9.

15. Urbano RV, Ventura LM, Miller MT, Brandt CT, Ventura B, Miller LM. Formação de oftalmopediatras no Brasil. An Fac Med Univ Fed Pernamb. 2005;50(2):119-21.

16. Kohnen $\mathrm{T}$. The value of surveys for cataract and refractive surgery. J Cataract Refract Surg. 2003;29(9):1647-8.

17. Brasil. Ministério da Educação. Sistema CNRM. Instituições $x$ programas x vagas. Disponível em: http://mecsrv04.mec.gov.br/sesu/

18. Instituto Brasileiro de Geografia e Estatística - IBGE. Você sabia? Curiosidades. Disponível em: http://www.ibge.gov.br/7a12/ voce_sabia/curiosidades/curiosidade.php?id_curiosidade $=114$

19. Conselho Brasileiro de Oftalmologia. Censo 2001: distribuição dos oftalmologistas por estado. São Paulo: CBO; 2001.

20. Accreditation Council for Graduate Medical Education. Ophthalmology Resident Operative Minimum Requirements. Available from: http://acgme.org/acWebsite/RRC_240/ 240_MinimumsOperativeTable.pdf

21. Brasil. Conselho Federal de Medicina. Resolução CFM n ${ }^{\circ}$ $1785 / 2006$, de 5 de abril de 2006. Dispõe sobre a nova redação do Anexo II da Resolução DFM n ${ }^{\circ} 1763 / 05$ que celebra o convênio de reconhecimento de especialidades médicas firmado entre o Conselho Federal de Medicina (CFM), a Associação Médica Brasileira (AMB) e a Comissão Nacional de Residência Médica (CNRM). Disponível em: http:// www.portalmedico.org.br/resolucoes/cfm/2006/ 1785_2006.htm.

\section{Endereço para Correspondência:}

Fundação Altino Ventura - FAV

Rua da Soledade, $n^{\circ} 170$, Boa Vista

CEP 50070-040 - Recife (PE), Brasil

Fone: (81)3302-4300

E-mail: fav@fundacaoaltinoventura.org.br camilaventura@hotmail.com 